\title{
High photon number path entanglement in the interference of spontaneously downconverted photon pairs with coherent laser light
}

\author{
Holger F. Hofmann* and Takafumi Ono \\ Graduate School of Advanced Sciences of Matter, Hiroshima University, \\ Kagamiyama 1-3-1, Higashi Hiroshima 739-8530, Japan
}

\begin{abstract}
We show that the quantum interference between downconverted photon pairs and photons from coherent laser light can produce a maximally path entangled $N$-photon output component with a fidelity greater than $90 \%$ for arbitrarily high photon numbers. A simple beam splitter operation can thus transform the 2-photon coherence of down-converted light into an almost optimal $N$-photon coherence.

PACS numbers: 42.50.Dv 03.67.Mn 03.65.Ud 42.50.Ar
\end{abstract}

One of the most challenging tasks of optical quantum information technologies is the generation and maintenance of entanglement in multi-photon states. Perhaps the most extreme case of $N$-photon entanglement is the path entangled state, where $N$ photons are in a superposition of being either all in one optical path or all in the other optical path inside a two path interferometer. In terms of the photon number states inside the interferometer, this state can be written as a superposition of two maximally distinguishable $N$-photon states,

$$
|\mathrm{NOON}\rangle=\frac{1}{\sqrt{2}}(|N ; 0\rangle+|0 ; N\rangle) .
$$

Since path entangled states represent a pure $N$-photon coherence, they are optimally sensitive to small phase shifts between the two optical paths. The generation of path entangled states with high photon number could therefore improve the precision of phase measurements to the fundamental quantum mechanical limit [1, 2, 3]. Various methods for generating path entangled states with arbitrary numbers of photons have been proposed [4, 5, 6, 7], but up to now, all known methods become more and more complicated as photon number increases, making it difficult to implement them for more than the three or four photon states already realized experimentally [8, 9]. Specifically, these experimental demonstrations were based on post-selection of the output, which means that only a very limited fraction of the actual $N$-photon emissions of the sources could contribute to the observed $N$-photon interferences. It is therefore of great interest to develop sources that provide $N$-photon path entanglement at high fidelity without requiring any post-selection of the output.

In the case of three photons, a particularly simple and elegant way to generate path entanglement is by an interference between coherent light and downconverted photon pairs at a beam splitter [10]. This method can be interpreted as a cancellation of all output components other than $|N ; 0\rangle$ and $|0 ; N\rangle$ by destructive quantum interference. Unfortunately, the exact cancellation of unwanted terms cannot be achieved at photon numbers higher than three, so that the extension to higher photon numbers appears to be difficult [11]. Nevertheless, it may be interesting to consider just how close we can get to ideal path entangled $N$-photon states by using only the basic two mode interference of downconverted photon pairs with laser light at a single beam splitter.

In this paper, we show that the interference between coherent laser light and downconverted photon pairs at a beam splitter can produce path entangled states of arbitrarily high photon number $N$ with fidelities greater than $90 \%$. In fact, the fidelity of the path entanglement increases with larger photon numbers $N$, approaching a maximum of $\sqrt{8 / 9}$ or $94.3 \%$ at very high $N$. The reason for this surprisingly strong non-classical effect is the combination of a perfect two photon coherence in the downconverted light with the lower photon number fluctuations of the coherent state of the laser. When the average photon numbers contributed by each field are nearly equal, this results in a slightly anti-squeezed photon number distribution around the maximum at $|N / 2, N / 2\rangle$, with a perfect multi-photon interference pattern "imprinted" upon it. By sending this multi-photon interference pattern "back" through a beam splitter, the multi-photon coherence is recovered and path entanglement results. The $N$-photon coherence observed in the interference between coherent laser light and downconverted photon pairs can thus be understood as a reversal of the interference process that is normally used to verify path entanglement.

A possible experimental setup for the observation of path entanglement in the interference of laser light and downconverted photon pairs is shown in fig:1. The quantum state of the input light is represented by a coherent state $|\alpha\rangle$ describing the laser light in input mode $\hat{a}$ and a squeezed vacuum state $|\gamma\rangle$ describing the downconverted photon pairs in input mode $\hat{b}$. The light interferes at the input beam splitter and passes through the two arms of a Mach-Zehnder interferometer. In the output, the $N$-photon coherence between the arms of the interferometer is 


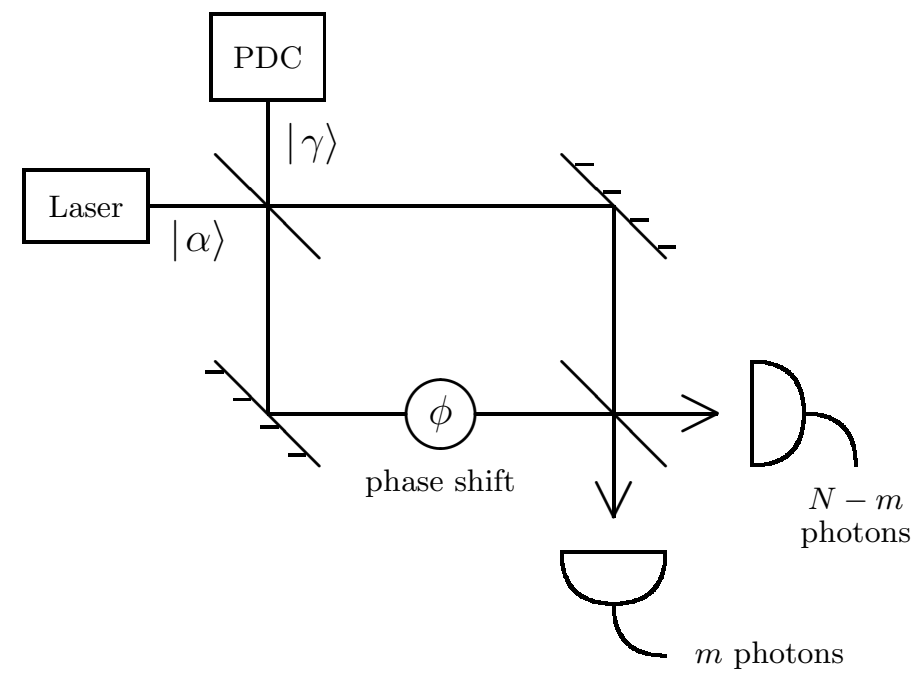

FIG. 1: Schematic setup for the observation of $N$-photon path entanglement in the interference between coherent laser light and photon pairs generated by parametric downconversion (PDC) using a Mach-Zehnder interferometer with a variable phase shift $\phi$. $N$-photon interference fringes can be observed in the output probabilities of the coincidences between $N-m$ photons in one output port and $m$ photons in the other output port.

observed in the phase dependence of the $N$-photon distribution between the two output modes. The key point about the $N$-photon interferences observed in the output is that it is not possible to distinguish the photons emitted by the laser from the photons generated by downconversion [12]. Therefore, each $N$-photon interference pattern is defined by a superposition of components $|N-2 k ; 2 k\rangle$ describing the emission of $N-2 k$ photons by the laser and the generation of $k$ photon pairs by parametric downconversion.

The coherent amplitudes of the components originate from the local coherences between the photon number states in each input mode. In the case of the coherent state $|\alpha\rangle$ describing the laser light in input mode $\hat{a}$ and the squeezed vacuum state $|\gamma\rangle$ describing the downconverted photon pairs in input mode $\hat{b}$, these local coherences can be represented by the effects of the respective annihilation operator on each state,

$$
\begin{aligned}
& \hat{a}|\alpha\rangle=\alpha|\alpha\rangle, \\
& \hat{b}|\gamma\rangle=\gamma \hat{b}^{\dagger}|\gamma\rangle .
\end{aligned}
$$

The quantum coherence of the normalized $N$-photon component $|\eta\rangle_{N}$ of the two mode light field can then be represented by combining the local coherences in such a way that the operators preserve the total photon number. A convenient representation of the $N$-photon coherence then reads

$$
\hat{a}^{\dagger} \hat{b}|\eta\rangle_{N}=\eta \frac{1}{N}\left(\hat{a}^{\dagger} \hat{a}\right) \hat{b}^{\dagger} \hat{a}|\eta\rangle_{N}, \quad \text { where } \quad \eta=\frac{N \gamma}{\alpha^{2}} .
$$

Significantly, the normalized $N$-photon state generated by the interference of laser light and downconverted light depends only on the ratio between the squeezing amplitude $\gamma$ and the squared coherent state amplitude $\alpha$. It is therefore possible to generate all of the interferometric states defined by eq. (3) with an arbitrarily fixed downconversion amplitude $\gamma$ by adjusting only the amplitude of the coherent laser light.

Eq.(3) uniquely defines the quantum state $|\eta\rangle_{N}$ and it is in principle no problem to derive the exact expansion into the input photon number basis $|N-2 k ; 2 k\rangle$. However, it is easier to understand the multi-photon coherence of the state by looking at the approximate solution in the limit of high photon numbers. It is then possible to simplify the effects of the annihilation and creation operators by using the approximation $\sqrt{n+1} \approx \sqrt{n}$. The relation between the amplitudes of the photon number components of $|\eta\rangle_{N}$ can then be approximated by

$$
\langle N-2(k+1) ; 2(k+1) \mid \eta\rangle_{N \rightarrow \infty} \approx \eta\left(1-\frac{2 k}{N}\right)\langle N-2 k ; 2 k \mid \eta\rangle_{N \rightarrow \infty} .
$$


By subtracting the amplitude of $k$ from the amplitude of $k+1$, the $k$ dependence of the amplitudes can be expressed in terms of a differential equation,

$$
\frac{d}{d k}\langle N-2 k ; 2 k \mid \eta\rangle_{N \rightarrow \infty} \approx-\frac{\eta}{N}\left(2 k-N\left(1-\frac{1}{\eta}\right)\right)\langle N-2 k ; 2 k \mid \eta\rangle_{N \rightarrow \infty} .
$$

This differential equation is solved by a Gaussian centered around $2 k=N(1-1 / \eta)$, with a variance of $N /(2 \eta)$ in $k$. At high photon numbers, the interferometric states can therefore be approximated by Gaussian two photon coherent states. For $\eta=2$, the maximal amplitude of the Gaussian state is located at $|N / 2 ; N / 2\rangle$, indicating equal numbers of photons contributed by each input mode,

$$
|\eta=2\rangle_{N \rightarrow \infty} \approx\left(\frac{4}{\pi N}\right)^{1 / 4} \sum_{k=0}^{N / 2} \exp \left(-\frac{(2 k-N / 2)^{2}}{2 N}\right)|N-2 k ; 2 k\rangle .
$$

To compare this interferometric state with the path entangled state given by eq.(1), we have to consider what kind of input state would result in ideal path entanglement inside the interferometer. We can do this by applying a beam splitter transformation to the photon number basis used in eq.(11). When the $|N ; 0\rangle$ component of this state is transformed by a beam splitter, this results in a binomial distribution of the photons between the two modes, with a maximal amplitude at $|N / 2 ; N / 2\rangle$. Quantum interference with the $|0 ; N\rangle$ component eliminates the components with an odd photon number in one of the modes. At high photon number, the path entangled state can therefore also be approximated by a Gaussian two photon coherent state centered at $|N / 2 ; N / 2\rangle$,

$$
|\mathrm{NOON}\rangle_{N \rightarrow \infty} \approx\left(\frac{8}{\pi N}\right)^{1 / 4} \sum_{k=0}^{N / 2} \exp \left(-\frac{(2 k-N / 2)^{2}}{N}\right)|N-2 k ; 2 k\rangle
$$

In fact, the only difference between the $\eta=2$ interferometric state given in eq.(6) and the path entangled state in eq.(7) is the variance of the Gaussian, which is twice as high for the $\eta=2$ state. In the limit of high photon number, the overlap between the maximally path entangled state and the $N$-photon component of the interference between coherent light and downconversion is thus equal to the overlap of two Gaussian states with a variance ratio of two,

$$
\left|\langle\mathrm{NOON} \mid \eta=2\rangle_{N \rightarrow \infty}\right|^{2} \approx \sqrt{\frac{8}{9}}
$$

At high photon numbers, we can therefore expect to find a maximally path entangled state with a fidelity approaching $\sqrt{8 / 9} \approx 0.943$, or $94.3 \%$.

Due to the high fidelity of the maximally path entangled state, the interference between laser light and downconverted photon pairs can produce high visibility $N$-photon interference fringes without any post-selection. This is a significant difference that distinguishes the present method of obtaining $N$-photon interference from previous experimental realizations [8, 9, 13, 14, 15]. To the best of our knowledge, the present method of obtaining $N$-photon interference fringes is the first method that can overcome the phase super sensitivity threshold discussed in [14, 15] at arbitrarily high photon number $N$ using only standard quantum optical technologies. A quick and efficient estimate of the $N$-photon visibility achieved by the $\eta=2$ state can be obtained by noting that the $\eta$ states are all orthogonal to the path entangled state with the opposite phase, $(|N ; 0\rangle-|0 ; N\rangle) / \sqrt{2}$. Therefore, the coherence between the $|N ; 0\rangle$ and the $|0 ; N\rangle$ components in the photon number basis of the paths inside the interferometer is equal to half the fidelity, and the visibility of the $N$-photon fringes will be equal to the fidelity. Since the phase sensitivity of an $N$ photon fringe with visibility $V$ is given by $1 /(\delta \phi)=V N$, we can expect a phase sensitivity of $0.94 N$ in the high photon number limit, a result that is only slightly lower than the Heisenberg limit of $N$ achieved by maximal path entanglement. It should also be noted that experimental errors presently limit multi-photon visibilities to much lower values. It is therefore likely that the small deviation of the $\eta=2$ state from the maximally path entangled state will not be the major factor limiting the phase sensitivity achieved in an actual experiment.

To confirm the validity of our approximations and their application to photon numbers as low as the experimentally realized four photon interferences, we have analyzed the precise overlap between the $\eta=2$ states and the corresponding path entangled states. This numerical analysis is based on the exact analytical forms of the quantum states given by

$$
\begin{aligned}
|\eta\rangle_{N} & =C_{\text {norm }} \sum_{k=0}^{N / 2} \frac{1}{k !} \sqrt{\frac{(2 k) ! N !}{(N-2 k) !}}\left(\frac{\gamma}{2 \alpha^{2}}\right)^{k}|N-2 k ; 2 k\rangle, \\
|\mathrm{NOON}\rangle_{N} & =\sum_{k=0}^{N / 2} \sqrt{\frac{N !}{(N-2 k) !(2 k) !}}\left(\frac{1}{\sqrt{2}}\right)^{N-1}|N-2 k ; 2 k\rangle,
\end{aligned}
$$




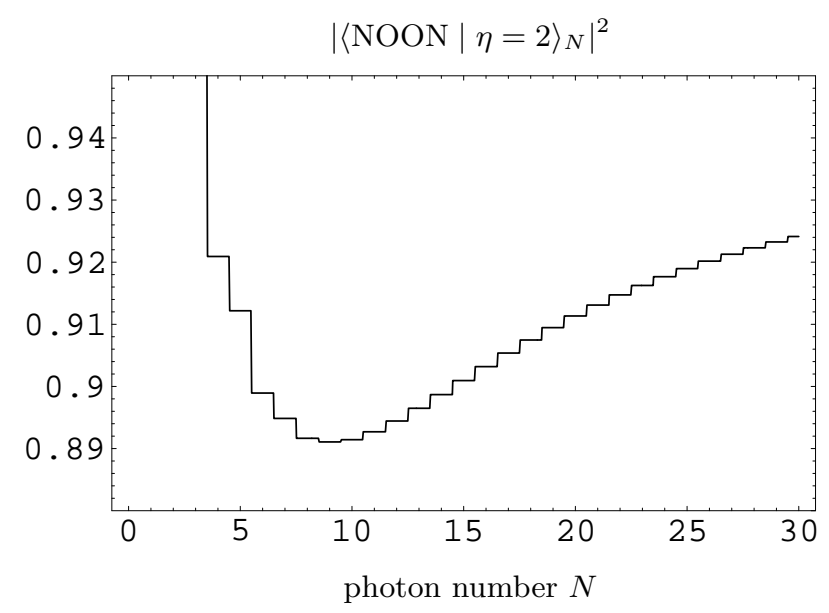

FIG. 2: Overlap between the $\eta=2$ interferometric state $|\eta=2\rangle_{N}$ and the ideal path entangled state $\mid$ NOON $\rangle_{N}$ as a function of total photon number $N$.

TABLE I: Optimized fidelities $F=\mid\left.\langle$ NOON $\mid \eta\rangle\right|^{2}$ for photon numbers ranging from $N=2$ to $N=15$. The result for $N=100$ is included as an illustration of the high photon number limit.

\begin{tabular}{c|ccccccccccccccc|}
\hline \hline$N$ & 2 & 3 & 4 & 5 & 6 & 7 & 8 & 9 & 10 & 11 & 12 & 13 & 14 & 15 & 100 \\
\hline$\eta$ & 2.00 & 3.00 & 2.31 & 2.48 & 2.36 & 2.36 & 2.32 & 2.30 & 2.28 & 2.26 & 2.24 & 2.22 & 2.21 & 2.19 & 2.02 \\
$F$ & $100 \%$ & $100 \%$ & $93.3 \%$ & $94.1 \%$ & $92.4 \%$ & $92.4 \%$ & $92.0 \%$ & $92.0 \%$ & $92.0 \%$ & $92.0 \%$ & $92.1 \%$ & $92.1 \%$ & $92.2 \%$ & $92.3 \%$ & $94.1 \%$ \\
\hline \hline
\end{tabular}

where $C_{\text {norm }}$ is the normalization factor of the interferometric state. Fig. 2 shows the overlap of the $\eta=2$ state with the maximally path entangled state as a function of total photon number $N$. Interestingly, the overlap has a minimum of $89.1 \%$ at $N=9$ photons. While this is already a rather high fidelity, it is possible to improve it somewhat by varying $\eta$. The results of this optimization for $N=2$ to $N=15$ and for $N=100$ are summarized in table 1 . These results indicate that fidelities of $92 \%$ or greater can be obtained at any photon number $N$.

Experimentally, the path entanglement of the $\eta=2$ interferometric state can be observed by conventional $N$-photon coincidence measurements, where the contribution of components greater than $N$ can be neglected if the amplitudes are sufficiently small. Since no post-selection is necessary, all $N$-photon emission events provide a valid measurement outcome, so the detection efficiency is limited only by the available photon counting technologies. The limitation of fidelity to between $92 \%$ and $94.3 \%$ should be a small price to pay for the resulting intrinsic efficiency of $100 \%$ for the interferometric method. In fact, previous experimental realizations of maximally path entangled states had much lower fidelities (as indicated e.g. by the raw data visibilities of $61 \%$ in [8] and $42 \%$ in [9]). It is therefore likely that the simple and efficient method of obtaining path entanglement presented here will outperform methods that could achieve maximal path entanglement if experimental conditions were ideal.

In practice, present downconversion methods are unfortunately still limited to rather low photon numbers. It will therefore be extremely difficult to observe coincidences of more than eight photons with a conventional setup. However, the validity of our results at arbitrarily high photon numbers indicates that $N$-photon path entanglement is in principle available whenever a squeezed vacuum interferes with laser light of an appropriate amplitude. If the technical problem of realizing precise photon number resolving measurements can be solved, it will therefore be possible to realize sources of path entanglement with intensities far greater than those presently feasible.

It may be worth noting that our analysis not only provides a new and more efficient method of generating path entanglement, it also illustrates an interesting fundamental relation between the two photon coherence observed in spontaneous parametric downconversion and vacuum squeezing and the $N$-photon coherence of path entanglement. Specifically, the interference pattern that is a typical feature of path entanglement is in fact a two photon coherence, as shown by the photon number expansions of the ideal path entangled states given by eqs. (7) and (10). Two 
mode interference at a beam splitter thus transforms $N$-photon path entanglement into two photon coherence and vice versa. This relation between two photon coherence and entanglement in optical quantum systems may have a a broader significance for the characterization of optical entanglement in terms of higher order coherences [16, 17, 18].

In conclusion, our results clearly show that $N$-photon path entanglement occurs naturally in the interference between coherent laser light and downconverted photon pairs. The high phase sensitivity of multi-photon path entanglement can therefore be achieved without any artificial tailoring of multi-photon interferences by post-selection or additional non-linear processes. Instead, it is sufficient to interfer the photon pairs generated in standard single mode downconversion with sufficiently attenuated laser light, so that the average photon numbers contributed by each source are approximately equal. By optimizing the ratio of coherent light and downconverted light, it should then be possible to obtain $N$-photon interference fringes with visibilities greater than $90 \%$ for any photon number $N$. The phase super-sensitivity of high photon number path entangled states is thus much easier to obtain than previously assumed.

Part of this work has been supported by the Grant-in-Aid program of the Japanese Society for the Promotion of Science and by the JST-CREST project on quantum information processing.

* Electronic address: h.hofmann@osa.org

[1] Z.Y. Ou, Phys. Rev. A 55, 2598 (1997).

[2] V. Giovannetti, S. Lloyd, and L. Maccone, Science 306, 1330 (2004).

[3] D. Bouwmeester, Nature (London) 429, 139 (2004).

[4] P. Kok, H. Lee, and J. P. Dowling, Phys. Rev. A 65, 052104 (2002).

[5] J. Fiurasek, Phys. Rev. A 65, 053818 (2002).

[6] G.J. Pryde and A.G. White, Phys. Rev. A 68, 052315 (2003).

[7] H.F. Hofmann, Phys. Rev. A 70, 023812 (2004).

[8] P. Walther, J.-W. Pan, M. Aspelmeyer, R. Ursin, S. Gasparoni, and A. Zeilinger, Nature (London) 429, 158 (2004).

[9] M.W. Mitchell, J.S. Lundeen, and A.M. Steinberger, Nature (London) 429, 161 (2004).

[10] F. Shafiei, P. Srinivasan, and Z. Y. Ou, Phys. Rev. A 70, 043803 (2004).

[11] B. Liu and Z. Y. Ou, Phys. Rev. A 74, 035802 (2006).

[12] An experimental demonstration of quantum interference based on the indistinguishability of photons from a coherent field and from downconversion has been reported in Y. J. Lu and Z. Y. Ou, Phys. Rev. Lett. 88, 023601 (2001).

[13] F. W. Sun, B. H. Liu, Y. F. Huang, Z. Y. Ou, and G. C. Guo, Phys. Rev. A 74, 033812 (2006).

[14] K. J. Resch, K. L. Pregnell, R. Prevedel, A. Gilchrist, G. J. Pryde, J. L. O’Brien, and A. G. White, Phys. Rev. Lett. 98, 223601 (2007).

[15] T. Nagata, R. Okamoto, J. O'Brien, K. Sasaki, and S. Takeuchi, Science 316, 726 (2007).

[16] G. S. Agarwal and A. Biswas, New J. Phys. 7, 211 (2005).

[17] E. Shchukin and W. Vogel, Phys. Rev. Lett. 95, 230502 (2005).

[18] M. Hillery and M.S. Zubairy, Phys. Rev. Lett. 96, 050503 (2006). 